\title{
Display System Using Wine Glass
}

\author{
Takumi Kobayashi, Toshiaki Yamanouchi, Kazuhisa Yanaka \\ Kanagawa Institute of Technology \\ 1030 Shimo-ogino, Atsugi-shi, Kanagawa-ken, 243-0292 Japan \\ ktamethyst279@gmail.com; yama@ic.kanagawa-it.ac.jp; yanaka@ic.kangawa-it.ac.jp
}

\begin{abstract}
Projection mapping is a technology that performs texture mapping by projecting light, including texture or image, onto an opaque real object with a projector. By applying this technology to plates and foods in restaurants, customers can enjoy not only the taste and flavor but also visual effects. However, projection mapping is difficult to apply to beverages and its glasses because they are usually transparent and reflect only a small amount of light. Hence, we developed a technology similar yet different from projection mapping. The main idea is to observe the image displayed on a flat panel display on the back through a non-flat transparent object such as a wine glass. When the wine glass with wine is observed diagonally from above, it appears to consist of three parts: the upper empty part, the water surface part, and the lower part filled with wine. Different animation is displayed in each part.
\end{abstract}

Keywords: Wine glass, Display, Mixed reality, Projection mapping.

\section{Introduction}

A new visual expression arises when a real object and light including image information are combined. A well-known example is projection mapping [1][2]. It is similar to texture mapping in CG applications, with the difference being that the real projector emits light containing texture to real objects. The texture may be produced through live-shot or computer graphics (CG) and the object includes a wide range of things, such as the exterior and interior of a building, furniture, and general goods. Artistic value is added in many cases. The history of the projection mapping dates back to the Singing Busts of the Haunted Mansion ride in Disneyland [1]. Later, with the appearance of Spatial Augmented Reality [3] and Shader Lamps [4], this technology attracted the attention of researchers.

In recent years, some restaurants have introduced projection mapping into the interior and food decoration. In dining, not only taste and flavor but also visual beauty, such as the decoration of food and plates, are important. Therefore, attempts have been made to add virtual decorations to wedding cakes via projection mapping [5]. In this case, projection mapping works well because most plates and the food on them are opaque. By contrast, beverages are often clear and the glasses used to hold them are also usually clear. Thus, projection mapping may be difficult to apply because glasses do not reflect a sufficient amount of light. Therefore, we developed a technology in which a display device such as a liquid crystal display (LCD) is placed behind a wine glass and the light emitted is refracted by the wine glass itself or the wine and reaches human eyes. This is similar yet different from projection mapping. The result is an amusing effect, such as an illusion of something appearing on the surface or in the wine.

\section{Method}

As shown in Figure 1, a wine glass is housed in a box to block unwanted light. This side and the other side of the box are open, and an LCD is placed behind the box. When the wine glass is viewed from the front and diagonally from above, one can see an empty part, a liquid surface part, and a lower part filled with liquid. A template (Figure 2) is created by tracing the outline of the glass and its three parts and then correcting the outline with Adobe Illustrator. When this template is displayed on the LCD, the ray emitted from each LCD pixel is refracted by the wine glass and reaches the eyes of the observer (Figure 3). Each color corresponds to a different part (Figure 4). 


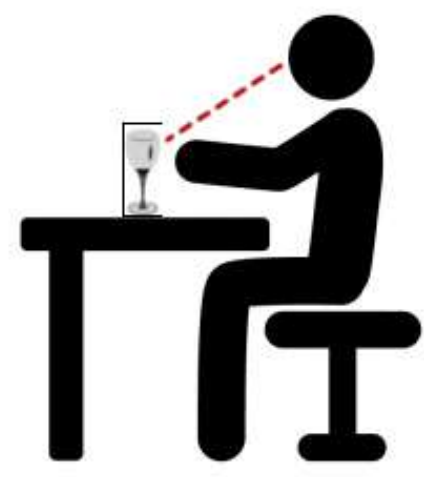

Fig. 1: Viewing condition.

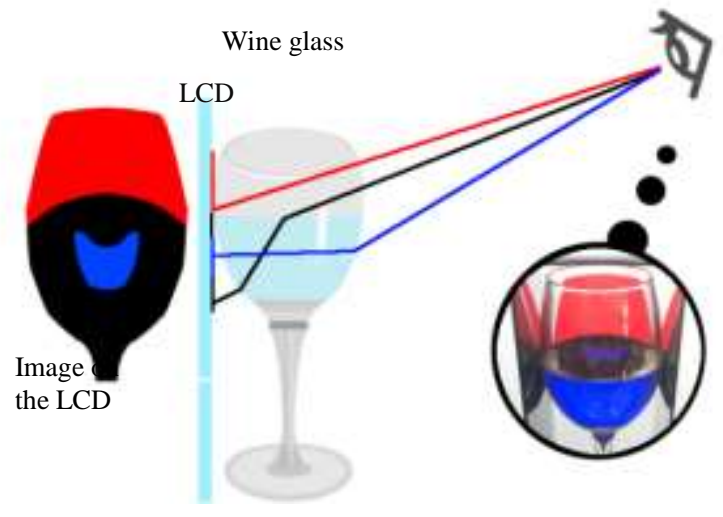

Fig. 3: Path of rays emitted from LCD pixels and refracted by the wine glass.

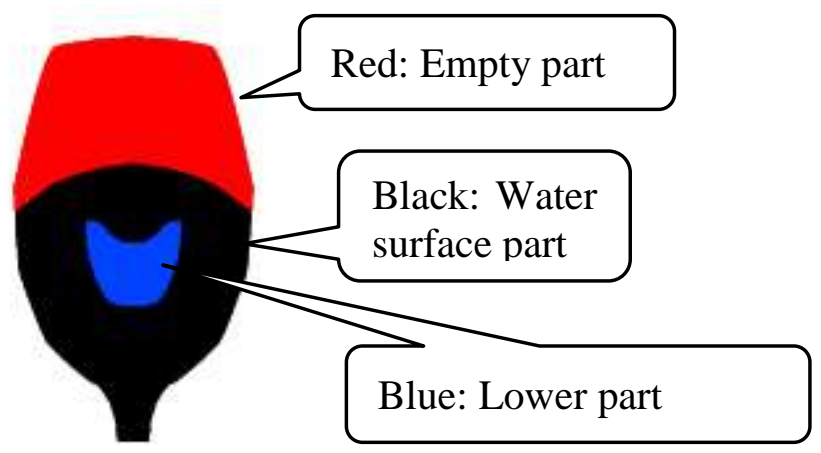

Fig. 2: Template.

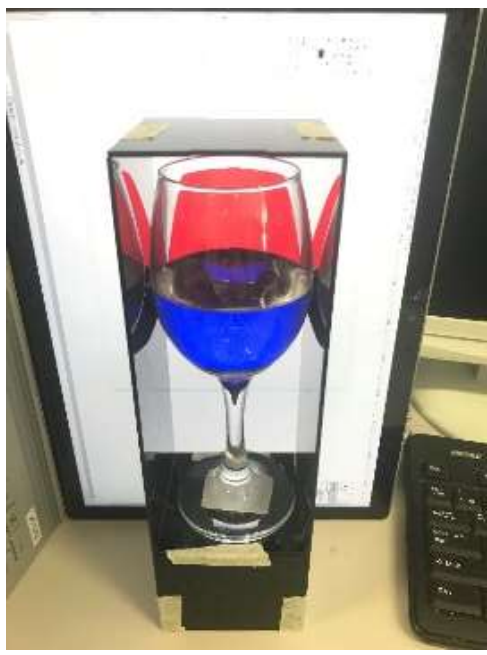

Fig. 4: Wine glass seen from the point of view of the observer.

In conventional projection mapping, a projector projects an image onto an opaque object and the reflected light is observed. In contrast, in our system, the light is emitted from a flat panel display placed on the back and refracted by a transparent object. Thus, projection mapping and our system complement each other.

\section{Experiment}

Figure 5 shows two kinds of video works, Work A and Work B, which were produced by combining three materials, each of which is based on the designed template. The produced image was displayed on the LCD situated behind a wine glass and observed from the front of the wine glass. Figure 6 shows the results. The displayed animation was attractive and presented an illusion showing various animated objects in the wine glass. 


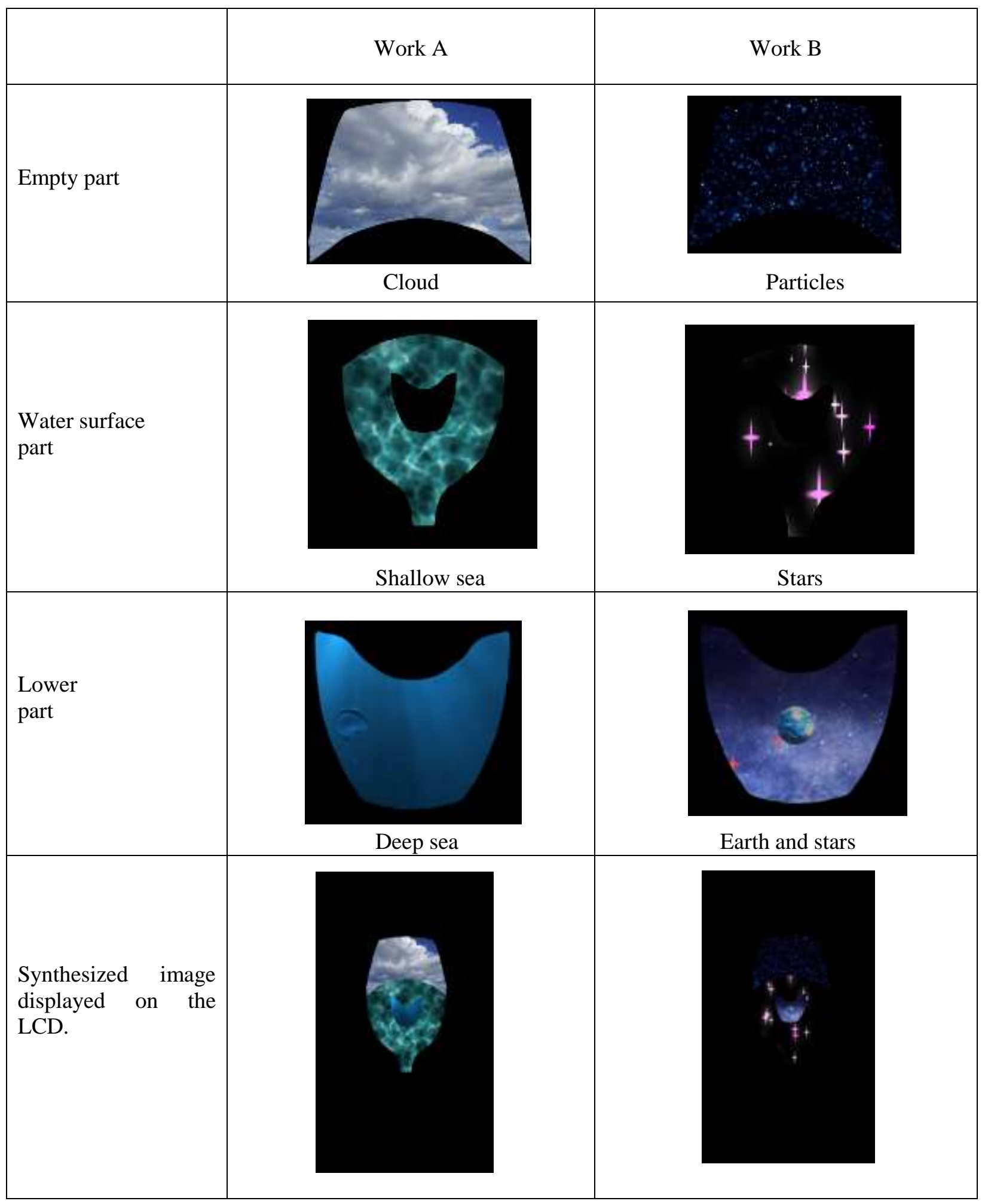

Figure 5: Creation of Work A and Work B. 

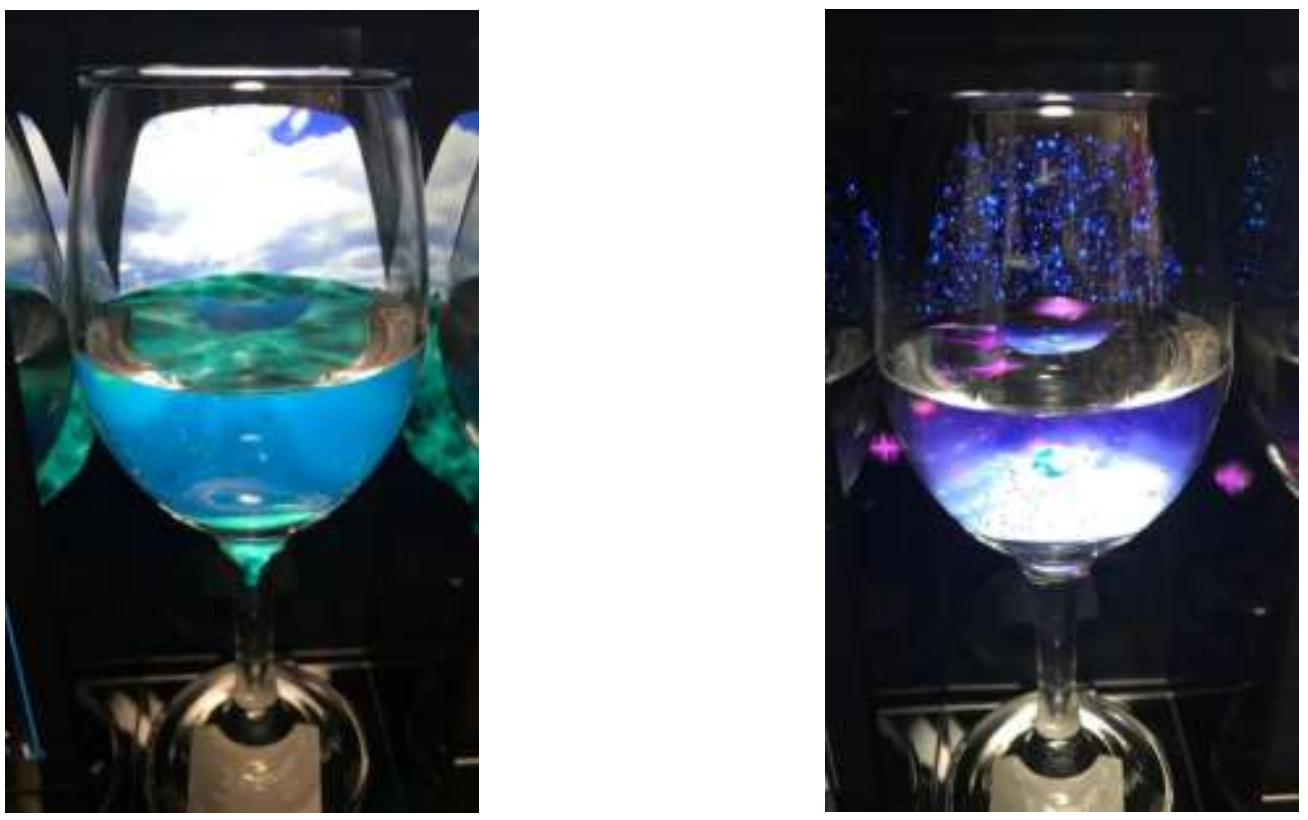

Fig. 6: Resulting display.

\section{Conclusion}

This study develops a new video display system that shows different animations on three parts of a wine glass, namely, the upper empty part, the surface of the wine, and the lower part filled with liquid. An LCD is placed behind a wine glass and is used to observe the wine glass from the front. This display system can be considered as a kind of a mixed reality system because it is a mixture of a real wine glass filled with real wine and virtually animated objects. The displayed animations are usually distorted because wine glasses are not made as precisely as optical components and the wine surface can sometimes be wavy. Furthermore, a limitation is that the observer can see the correct image only from a specific viewpoint (front, diagonally above the wine glass). Nevertheless, the results bring out unique feelings for observers.

Currently, only cocktails offer aesthetic appeal in the real world. If the proposed system is put into practical use, wines could also be presented with the same aesthetic value. The video does not disappear even when the glass is held because the video is displayed from the back rather than in front of the glass. This system allows the wine to be enjoyed through taste and sight. In the future, this technology could be applied to something other than wine glasses.

\section{References}

[1] PMC - Projection Mapping Central, The Illustrated History of Projection Mapping [Online]. Available: http://projection-mapping.org/the-history-of-projection-mapping/

[2] Oliver Bimber, Ramesh Raskar, Spatial Augmented Reality Merging Real and Virtual Worlds, A K Peters, Ltd, 2005. [Online]. Available: http://pages.cs.wisc.edu/ dyer/cs534/papers/SAR.pdf

[3] Ramesh Raskar, Greg Welch, Henry Fuchs, "Spatially Augmented Reality," First International Workshop on Augmented Reality, San Francisco, November 1, 1998.

[4] Ramesh Raskar, Greg Welch, Kok-Lim Low, Deepak Bandyopadhyay, "Shader Lamps: Animating Real Objects With Image-Based Illumination," Eurographics Workshop on Rendering, London, England, June 25-27, 2001.

[5] Facade Signage, Wedding Cake Projection Mapping: Ready, Steady, Animate. [Online]. Available: https://www.videomappingsoftware.com/blog/wedding-cake-projection-mapping/ 